\title{
Influence of NLTE calculations on the hydrogen lines in chromospheric models
}

\author{
B. Fuhrmeister ${ }^{1}$, C. I. Short ${ }^{2}$, and P. H. Hauschildt ${ }^{1}$ \\ 1 Hamburger Sternwarte, University of Hamburg, Gojenbergsweg 112, 21029 Hamburg, Germany \\ 2 St. Mary's University, Halifax, NS B3H 3C3, Canada \\ e-mail: bfuhrmeister@hs.uni-hamburg.de
}

Received 7 July 2005 / Accepted 3 March 2006

ABSTRACT

\begin{abstract}
We present extensive NLTE calculations for a semi-empirical solar 1D chromosphere model based on the VAL C model. We report on a significant influence of nitrogen, oxygen, and sulfur on the emergent hydrogen emission with respect to LTE vs. NLTE calculations for these elements. Moreover, we present a model spectrum with 20 light and iron group elements computed in NLTE. We compare this to an observed solar spectrum and to a photospheric model spectrum. We find that the agreement for this model with the data is less good than the original VAL C model especially in the UV. This may imply the need of changes in the chromospheric temperature structure or may point to general problems of static 1D chromospheric models.
\end{abstract}

Key words. line: formation - Sun: chromosphere

\section{Introduction}

The chromosphere is a thin layer in the outer atmosphere of latetype stars. In the classical picture the chromosphere is the place where the temperature of the atmosphere starts to rise outwards steepening in the transition region (TR) until it finally reaches coronal temperatures. This temperature rise induces chromospheric and TR emission which can be used to model the temperature distribution semi-empirically which was done for the Sun by e.g. Vernazza et al. (1981); Fontenla et al. (1990), for other active dwarf stars (e.g. Short \& Doyle 1998; Mauas \& Falchi 1994), and for red giants (e.g. Harper 1992). This picture of a spatially uniform temperature rise has been challenged by the discovery of CO molecules in the Sun's chromosphere (Ayres \& Testerman 1981) and other stars' chromospheres (Wiedemann et al. 1994). A more dynamical scenario pictures the chromosphere as a place where heating takes place in acoustic shock waves that are embedded in a cool plasma. Hydrodynamic calculations of the solar chromosphere have been performed in 1D (Carlsson \& Stein 1997, 2002), as well as in 3D (Wedemeyer et al. 2004). In any case the conditions in the chromosphere are more extreme than in the photosphere and therefore NLTE effects gain in importance. Very few extensive NLTE chromospheric studies have been carried out, though. Even for the Sun's photosphere, extensive NLTE line calculations have been carried out only recently (Short \& Hauschildt 2005). Fuhrmeister et al. (2005) found for M dwarfs that the NLTE treatment of C, $\mathrm{N}$, O has a significant influence on the amplitude of the hydrogen emission lines. The hydrogen spectrum is important not only as spectral diagnostic, but because the hydrogen ionization balance significantly influences the electron pressure in large parts of the atmosphere. It is also known that hydrogen pumps certain O I lines via an overlapping transition with $\operatorname{Ly} \beta$ (Athay \& Judge 1995; Carlsson \& Judge 1993). Also there are pumping effects between $\mathrm{Ly} \alpha$ and various Fe II, Ca II and $\mathrm{H}_{2}$ lines (McMurry et al. 1999).
The goal of this paper is to further investigate pumping effects between the veil of emission lines and the Ly continuum. We compute our models with the general purpose atmosphere code PHOENIX (Hauschildt et al. 1999) which is well suited for this purpose, because it is capable of allowing for millions of atomic and molecular background lines in LTE as well as for multi-level NLTE calculations for many species at the same time. We will investigate the influence of the LTE background lines that have been computed for the first time with high resolution under consideration of the chromospheric and TR temperature rise. We will take a special interest in the influence of nitrogen, oxygen, and sulfur on the line formation of hydrogen. We also present extensive multi-level NLTE chromospheric calculations on the basis of the VAL C model (Vernazza et al. 1981). We find that especially the Lyman continuum is quite sensitive to different NLTE sets used and that the agreement with the data is normally less good than for the original VAL C model, which was constructed to fit the data. Therefore, the temperature structure of the VAL C model would have to be altered in the TR and high chromosphere for large NLTE sets. Nevertheless, we think, that our NLTE models are more physical than the original VAL C model, since they account for EUV lines in the chromosphere and transition region and compute much more atomic transitions in NLTE than the VAL C model did. We will show a significant (NLTE) influence of ionised species from the transition region on the spectrum. This lines in with the results from Fontenla et al. (1990), who found that the correct treatment of the hydrogen ionisation - accounting for ambipolar diffusion in the lower transition region is important, but did not include other species than hydrogen in their calculations.

Our paper is structured as follows: we describe the assumptions made for the computations in Sect. 2 and present the comparison between various different LTE vs. NLTE models in Sect. 3. We discuss several aspects of the influence of the NLTE calculations in Sect. 4. 
Table 1. Important parameters of the chromospheric models used. Besides the model name it is indicated if LTE background lines are included in the model. The third column indicates which elements are treated in NLTE besides hydrogen, helium and calcium, with "large" meaning the NLTE set with 82 species as descried in Sect. 2.2. The fourth column indicates whether the opacity shortward of $2000 \AA$ is calculated on the 2 or $0.02 \AA$ wavelength grid.

\begin{tabular}{cccc}
\hline \hline Model name & LTE backgr. & NLTE species & gridding \\
\hline NOL & no & - & 2 \\
ATLF & yes & - & 0.02 \\
NOS & yes & N, O, S & 0.02 \\
LAR & yes & large & $\sim 0.02$ \\
LARN & no & large & $\sim 0.02$ \\
LAF & yes & large w/o second. lines & $\sim 0.02$ \\
LAFN & no & large w/o second. lines & $\sim 0.02$ \\
LAA & yes & large with Ar IV & $\sim 0.02$ \\
LAAN & no & large with Ar IV & $\sim 0.02$ \\
\hline
\end{tabular}

\section{Computational method}

The models calculated here are 1D chromospheric models with a chromospheric and TR temperature rise attached to the photosphere. All computations are done using the atmosphere code PHOENIX (Hauschildt et al. 1999). For solving the radiative transfer and the statistical equilibrium equations in PHOENIX an approximate $\Lambda$-operator iteration scheme is applied, which can solve the equations for many species simultaneously (Hauschildt 1993).

One drawback in PHOENIX regarding chromospheric calculations is that presently no partial frequency redistribution (PRD) is included in the code; all calculations are done assuming complete frequency redistribution (CRD). This affects the line shapes and intensities of e.g. Ly $\alpha$ and Ly $\beta$ as well as some metal lines like $\mathrm{Mg}$ II $\mathrm{h}$ and $\mathrm{k}$. The influence of PRD vs. CRD on the Lyman lines has been studied for the Sun by e.g. Hubeny \& Lites (1995). They find an influence on the population of the excited and ionised states of hydrogen, while the influence on the population of the ground state is only very modest. The largest influence they find is on the wings of Ly $\alpha$. Since we do not study the Ly $\alpha$ line itself, the CRD treatment should not influence our results in a fundamental way.

\subsection{Atmospheric models}

Since we want to compare our data to the photospheric model from Short \& Hauschildt (2005) we used the same parameter of $T_{\text {eff }}=5777 \mathrm{~K}$ and $\log g=4.4377$. As chromospheric model we used the classical VAL C model (Vernazza et al. 1981). We used the temperature vs. column mass structure with the original turbulent velocity distribution of this model and recomputed the model properties such as $p_{\text {gas }}, p_{\mathrm{e}}$, and the NLTE departure coefficients self-consistently in PHOENIX. The different models we investigated here are distinguished mainly by the treatment of the atomic background lines and the different sets of species treated in NLTE. Table 1 gives an overview over the different models used here. In general a line may either be treated in NLTE, in LTE or it may be omitted. To calculate lines of a certain species in NLTE one has to specify a model atom which contains a selection of lines to be treated in NLTE. For our PHOENIX models every available line and therefore virtually all lines of the given specified NLTE species are calculated in NLTE (see comment on the secondary lines in Sect. 4). Lines of non-LTE species are calculated in LTE for the models including

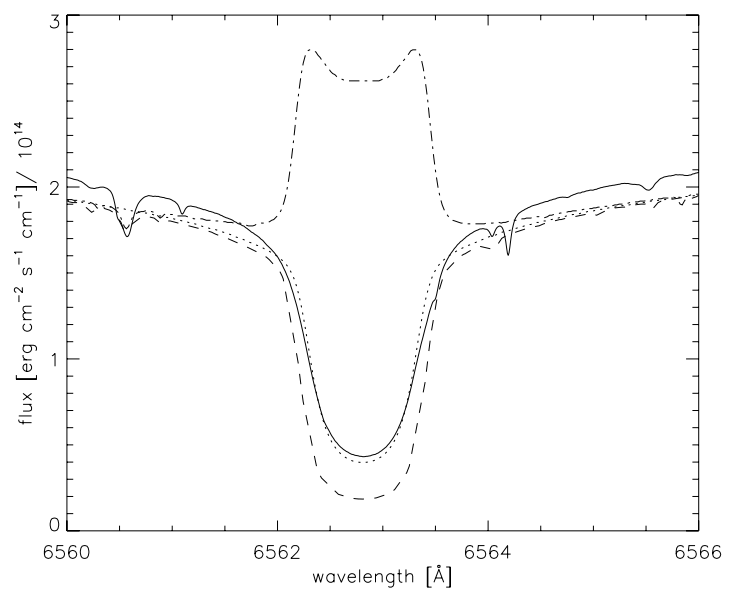

Fig. 1. Comparison of observed flux in $\mathrm{H} \alpha$ from the Neckel atlas (Neckel 1999) with our NOL model (dotted), our ATLF model (dotdashed), and our LARN model (dashed). The NOS model is nearly identical to the NOL model.

LTE background lines (ATLF, NOS, LAR, LAF, and LAA) or are omitted (NOL, LARN, LAFN, LAAN).

For comparison to solar data we used the Neckel atlas (Neckel 1999) in the optical and SUMER data (Curdt et al. 2001) in the UV. Redwards of $6000 \AA$ the photospheric model flux has to be increased by about four percent to match the data. The same is true for the chromospheric models. This offset in the red band can be seen in the far wings of $\mathrm{H} \alpha$ in Figs. 1 and 3. Note that the photospheric and chromospheric model intensity will join further away from line center of $\mathrm{H} \alpha$. A possible explanation for this offset in the red band is the Fe I over-ionisation in NLTE models with respect to LTE models. This allows more flux to escape in the UV resulting in less back-warming and less flux redistribution from the UV to the red band. PHOENIX LTE models do not show this problem.

For our computations we used the solar abundances from Grevesse \& Noels (1993). For our LARN model we also applied the original abundances from the VAL model (Vernazza et al. 1973) and found no significant deviation besides in individual metal lines.

\subsection{NLTE calculations}

All our models are computed with at least hydrogen, helium and Ca I-III in NLTE, since hydrogen and helium are the most abundant species in most parts of the considered atmosphere and the $\mathrm{Ca}$ II $\mathrm{H}$ and $\mathrm{K}$ line are important diagnostic lines. The data used for these and additional elements calculated in NLTE are taken from the Kurucz database (Kurucz \& Bell 1995) or from the CHIANTI database (Young et al. 2003) in the case of He I, II, O VI-VIII, N VII, C V, VI, and Ne II-X. Our NLTE calculations take into account all available levels for each considered ionisation stage. For the large NLTE sets we treated most of the light elements up to $\mathrm{Ca}$ as well as the iron group elements in NLTE summing up to 82 different ionisation stages and hundred thousands of lines. Specifically we have computed in NLTE all ionisation stages of $\mathrm{H}, \mathrm{He}, \mathrm{N}, \mathrm{O}$ and $\mathrm{Ne}$, the ionisation stages I and II of $\mathrm{Li}, \mathrm{Ti}$, and $\mathrm{Mn}$, the ionisation stages I to III of $\mathrm{Ca}$ and $\mathrm{Co}$, the ionisation stages I to IV of $\mathrm{C}, \mathrm{Na}, \mathrm{Mg}, \mathrm{Al}, \mathrm{Si}, \mathrm{K}, \mathrm{P}, \mathrm{Ni}$, and $\mathrm{Fe}$, and the ionisation stages I to VI of S. This ensures that the most important species in each layer of the model atmosphere are computed in NLTE with the exception of the high ionisation stages of carbon (see Sect. 4). 


\subsection{Background line opacity}

Our calculations take into account the veil of atomic background lines throughout the atmosphere, i.e. including the chromospheric and TR temperatures. Our wavelength resolution for these LTE lines regarding the radiative transfer in the models is $2 \AA$ for wavelengths shorter than $30000 \AA$ and normally $0.02 \AA$ shortward of $2000 \AA$, which will lead to sampling of every LTE line in the Lyman continuum. The NOL model and the models with large NLTE sets have a wavelength grid of $2 \AA$ shortward of $2000 \AA$ but the NOL model can be nevertheless compared to the ATLF model, since it includes no lines at all in the Lyman continuum. NLTE calculations lead to the insertion of additional wavelength points for every NLTE line leading to a non-regularly spaced wavelength grid. For the models with large NLTE sets the mean spacing is $0.01 \AA$ in the Ly continuum with about 15 percent of the spaces larger than $0.02 \AA$ but only 3 percent of the spaces larger than $0.04 \AA$. These models therefore have an opacity sampling equivalent to the fine wavelength grid.

\subsection{Flux contribution functions}

As a further diagnostic tool we incorporated the output of a disc integrated intensity contribution function in PHOENIX. The intensity contribution function $\mathscr{C}_{I}$ is normally defined on a $\log \tau_{0}$ scale, but since our chromospheric models are computed on a column mass scale, we computed also

$$
\mathscr{C}_{I}(\log m)=\mu^{-1} \ln 10 m \kappa S \mathrm{e}^{-\tau / \mu}
$$

with $S$ being the source function, $m$ the column mass, $\kappa$ the absorption coefficient, $\tau$ the optical depth and $\mu=\cos \theta$ the angle between the considered direction and the normal to the surface. This intensity contribution function is dependent on the considered direction and can be computed for any angle $\theta$. Additionally, we calculate the disc integrated value $\mathscr{C}_{F}$. Since we regard only models of the average quiet Sun here the disc integrated value is appropriate. The usage of the flux contribution function instead of the line depression contribution function as introduced by Magain (1986) is a legitimate approximation, since the considered lines are strong.

\section{Results}

\subsection{Influence of NLTE calculations}

The VAL C model recomputed with PHOENIX with hydrogen, helium and Ca I - III in NLTE and no background lines at all (our NOL model) gave for the $\mathrm{H} \alpha$ line very similar results as the original VAL $\mathrm{C}$ model and reproduced the observed $\mathrm{H} \alpha$ line quite well (see Fig. 1). An inclusion of all available atomic LTE background lines in the model calculations (our ATLF model) led to $\mathrm{H} \alpha$ in emission, though, which is caused by the atomic lines computed in LTE. Though LTE is not a valid assumption for the chromosphere, one can investigate, which elements cause the discrepancy in the Balmer lines (and have to be computed in NLTE therefore) and which elements have no influence on other elements than themselves. Therefore, we started to switch the elements on one by one for our NOL model, excluding the switched off elements totally from the calculations by turning down their abundance to zero. This shows that the major influence on the $\mathrm{H} \alpha$ is that of oxygen, nitrogen, sulfur and argon. Since there is no atomic data of argon available at the moment, we computed our NOS model, which is nearly identical to the

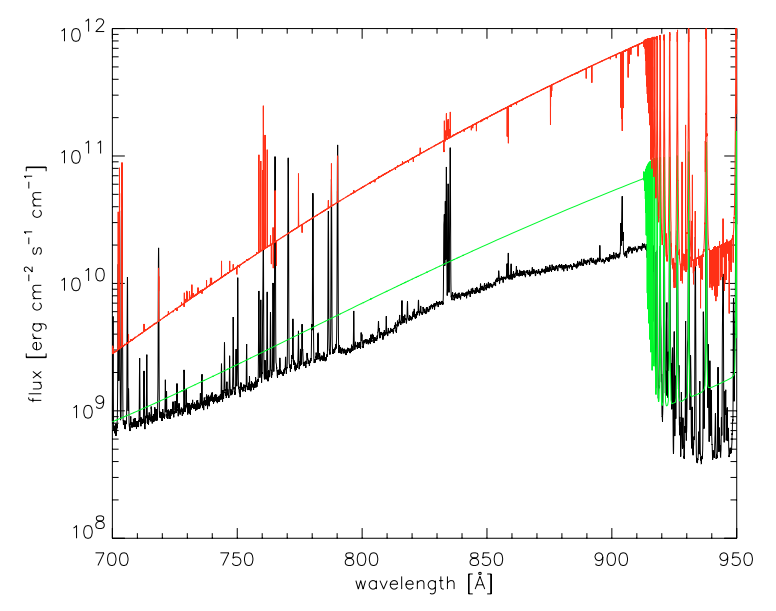

Fig. 2. Comparison of observed flux in the Lyman continuum from the SUMER atlas (Curdt et al. 2001) (black) with our NOL model (light grey/green) and our LARN model (grey/red).

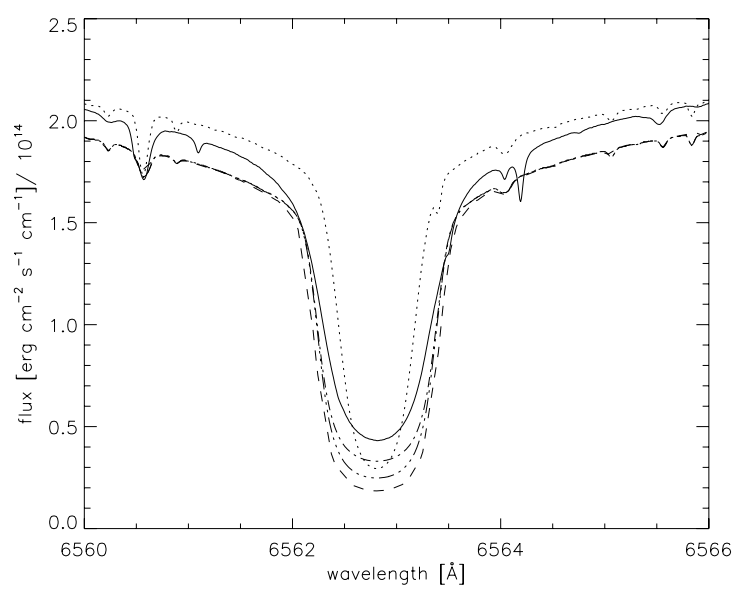

Fig. 3. Comparison of solar data (solid curve) for the $\mathrm{H} \alpha$ line to the photospheric model from Short \& Hauschildt (2005) (dotted), to our LAR model (dot-dashed), our LARN model (dashed), and our LAA model (triple-dot-dashed).

NOL model for the $\mathrm{H} \alpha$ line. Computing additional species in NLTE only lead to small changes and deepens the line further. Computing large NLTE sets and no additional LTE lines at all leads to the deepest line depression for the $\mathrm{H} \alpha$ line (LARN and LAAN models). This can be seen in Fig. 1.

In the UV part of the spectrum and especially in the Lyman continuum the influence of NLTE calculations is even more extreme. A comparison between our NOL model and SUMER data can be found in Fig. 2 showing again a good description of the data (within a factor of two). Inclusion of LTE or NLTE lines gives a significant higher flux level in the Lyman continuum. The intensity of the NOS model had to be scaled by a factor of 90 downwards to match the SUMER data, while the intensities of the LAR and the LARN model needed scaling by about 40 and 20, respectively. This strong dependence of the intensities in the Lyman continuum on the computation of LTE versus NLTE lines is due to pumping effects by strong lines out of which the most important influence is by the numerous strong lines of oxygen, nitrogen, sulfur, and argon. Other elements like the abundant carbon show only few and weaker lines in the Lyman continuum and have therefore less influence.

Compared to the NOL model this additional line flux of NLTE lines but especially of over-predicted LTE lines leads to 
more photo-ionisation events of hydrogen. The increased hydrogen ionisation reduces the Lyman continuum opacity and therefor allows more flux to escape there. Also the Balmer and Paschen lines are deeper for our NLTE models compared to the NOL model and all these lines go eventually into emission for the LTE ATLF model making it a probable explanation that there is also enhanced recombination and subsequent cascading down for hydrogen. Also, the NOL model has the lowest flux level in the Lyman continuum, since there is no photo-ionisation by any metal lines. Inclusion only of NLTE lines gives a significant increase, as these lines start to photo-ionise hydrogen. An inclusion of LTE background lines strengthens this effect due to the unrealistic high flux in these lines even for large NLTE sets.

To illustrate this, we compared the radiative absorption rates of the ground level of hydrogen to the ionisation state and found that they are lowest for the NOL model, and a factor of 5 and 100 higher for the LARN and the LAR model, respectively.

One attempt to deal with the metal lines is normally not to compute them in pure LTE but to turn them into scattering lines in a statistical way. This can be done in PHOENIX by choosing the parameter $\varepsilon$ in the relation $S=\varepsilon B+(1-\varepsilon) J$. $\varepsilon$ is chosen for the whole atmosphere, though and is not height dependent as for other codes. To test the influence of $\varepsilon$ we chose $\varepsilon=10^{-4}$, which should be typical for the upper chromosphere and TR (Anderson 1989) and recomputed our ATLF model. The result in the Lyman continuum is nearly identical to the NOL model and also represents the data well.

Besides these differences in spectral features, we have compared the electron and gas pressure of our models to that of the VAL model. While the gas pressure of our LARN and NOL model is nearly identical, it deviates from that of the VAL C model by up to 50 percent around the onset of the TR, but less than 10 percent in the rest of the atmosphere. The electron pressure deviates most in the mid chromosphere. The deviations of the NOL model to the VAL model are up to a factor of 2, while for the LARN model the deviations to the VAL model are up to a factor of 12. These changes in electron pressure lead to changes in the opacity and therefor to changes in the emergent intensities.

Therefore we conclude that the more realistic NLTE description of the metal lines calls for an alteration of the temperature structure of the original VAL model to fit the UV data again. Moreover, the strong influence of the NLTE computations may point to other model inadequacies and may demand a more realistic description with, for example, horizontal inhomogeneities.

\subsection{Comparison to a photospheric model}

We compared our LAR and LARN models, which we regard as our most realistic models, to the NLTE photospheric model of Short \& Hauschildt (2005). For the $\mathrm{H} \alpha$ line the comparison between models and data can be found in Fig. 3. Our LAR model is in good agreement with the data, though it produces slightly too less flux in the $\mathrm{H} \alpha$ line, while the chromospheric model not including the LTE background lines produces even less flux. Note that the chromospheric models provide a better fit of the width of the $\mathrm{H} \alpha$ line. This broadening is partly due to the chromospheric structure itself, but also partly due to the inclusion of additional micro-turbulence.

Another wavelength range, where the chromospheric models give a significant better overall fit, is the region around the $\mathrm{Ca}$ II $\mathrm{H}$ and $\mathrm{K}$ line (see Fig. 4). While the photospheric model does not provide a good fit for the wavelength region between the $\mathrm{Ca}$ II $\mathrm{H}$ and $\mathrm{K}$ line without scaling the intensity of the model

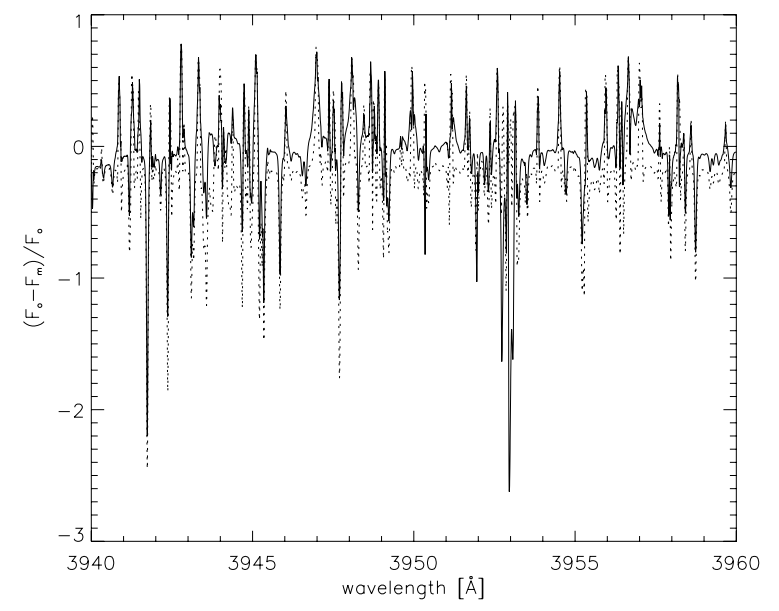

Fig. 4. Relative difference between the observed Flux $\left(F_{\mathrm{o}}\right)$ and the model flux $\left(F_{\mathrm{m}}\right)$ as a percentage of $F_{\mathrm{o}}$ for a wavelength range between the $\mathrm{Ca}$ II $\mathrm{H}$ and $\mathrm{K}$ lines. The solid line denotes our LAR model, while the dotted line denotes the photospheric model from Short \& Hauschildt (2005). It can be seen that the intensity of the photospheric model in average is about 10 percent too strong.

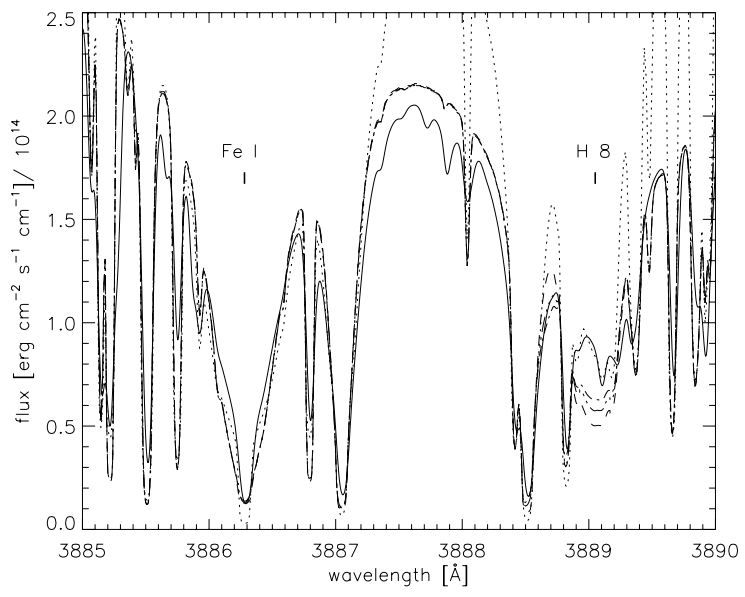

Fig. 5. Same as in Fig. 3 but for a wavelength range in the very blue part of the optical range including H8, where the chromosphere also influences the quasi-continuum. Again we compare solar data (solid curve) to the photospheric model (dotted), the LARN model (dashed), the LAR model (dot-dashed), and the LAA model (triple-dot-dashed).

for about 10 percent, the intensity of our LAR model fits without rescaling.

As another representative example of chromospheric influence, we present a wavelength range around $\mathrm{H}_{8}$ at $3889.05 \AA$. It includes further a strong Fe I lines at $3886.28 \AA$ and two less strong Fe I lines at 3887.05 and $3888.51 \AA$. The comparison can be found in Fig. 5. Note that the flux of the photospheric model is about 20 percent too strong, while the flux of the chromospheric model matches the data. The flux of the chromospheric model is significantly weaker than the flux of the photospheric model in the wavelength range between 2000 and $5000 \AA$.

\section{Discussion}

Even for the set with 82 NLTE species the two models with and without inclusion of the LTE background lines (LAR/LARN) are not identical in the hydrogen Balmer lines, though the difference decreases with increasingly larger NLTE sets. Therefore there must be still background lines being present which disturb the 
hydrogen ionisation balance. Since we found in the LTE calculations that also argon has an influence on the hydrogen lines, argon should be treated in NLTE, but unfortunately there is a lack of atomic data for argon among both the Kurucz and CHIANTI data. For the lower ionisation stages of argon only Ar IV is found in the CHIANTI database. An inclusion of Ar IV in the NLTE calculations decreases the difference between calculations with and without LTE background lines. From LTE line lists we know that there are also strong Ar V lines found in the Ly continuum, therefore, an inclusion of Ar V should improve the situation even more.

Nevertheless, our NLTE calculations sampled most of the strong observed lines in this wavelength region and therefore the treatment without LTE background lines gives the more realistic description of this region. For wavelength redwards of $\operatorname{Ly} \alpha$ the differences between the LAR and the LARN model spectrum decreases, though.

PHOENIX treats very weak lines of species computed in NLTE, nevertheless, in LTE. For a detailed description of how these secondary lines are treated, see Hauschildt \& Baron (1995). Though the secondary lines are not expected to alter our results, we checked their influence. A comparison of our models LAF and LAR and our models LAFN and LARN showed no significant differences for the $\mathrm{H} \alpha$ or $\mathrm{Ly} \alpha$ line or the Ly continuum besides in single metal lines.

Since carbon is a highly abundant species we originally tried to include all ionisation stages in our NLTE calculations, but it turned out that models that include C VI are not converging but varying randomly from iteration to iteration. This destabilization of the model is due to the coarse temperature sampling in the outermost layers of the model where C VI is very abundant only in the second outermost layer. The partial pressures undergo an increase of seven magnitudes for this layer. We conclude that for a proper NLTE treatment of C VI a finer temperature spacing in the TR is needed. Since C V has no impact on the $\mathrm{H} \alpha$ line we decided to compute the large NLTE set only with C I to IV.

To show the influence of the LTE treatment of certain species on properties of the radiative transfer we calculated the flux contribution function for the LAR, the LARN and the NOS model. In Figs. 6 and 7 we show the monochromatic source function $S_{v}$, the Planck function $B_{v}$, the intensity $J_{v}$, and the flux contribution function $C_{\mathrm{F}}$ for our LARN and NOS models. Here $S_{v}$ is the total source function, i.e. $S_{v}$ is the ratio of the continuum plus line emission coefficient and the continuum plus line absorption coefficient. In the case without background line blanketing (our LARN model) $S_{v}$ follows $J_{v}$ and is only increased by the contribution of $B_{v}$ for layers in the transition region - which is above the peak of $C_{\mathrm{F}}$. This looks very similar for the LAR model, with the peak of $C_{\mathrm{F}}$ moved to slightly deeper layers in the atmosphere and $S_{v}$ starting to deviate slightly from $J_{v}$. Both effects are increased for the NOS model, which we show therefore here. The less deep depression of the $\mathrm{H} \alpha$ line for the NOS model compared to the LARN model is due to the formation in slightly deeper layers, where the source function can contribute more flux to the line. Also $S_{v}$ deviates from $J_{v}$ in the contributing layers.

From this example of the $\mathrm{H} \alpha$ line it can be seen that treating many background lines in LTE causes an unrealistic coupling between $S_{v}$ and $B_{v}$. Therefore, $S_{v}$ is too large in the outer atmosphere with the result that the $\mathrm{H} \alpha$ line is too strong in emission. To prevent the LTE lines from being over-thermalized, one can turn them artifically into scattering lines.

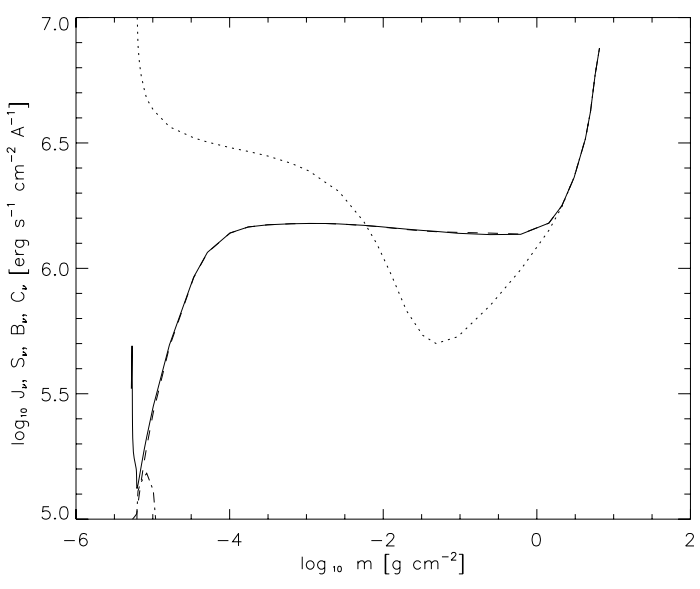

Fig. 6. Various radiative transfer quantities for the central wavelength of $\mathrm{H} \alpha$ for our LARN model. The solid curve is $S_{v}$, the dotted curve is $B_{v}$, the dashed curve is $J_{v}$, and the dash-dotted curve the scaled $C_{\mathrm{F}}$.

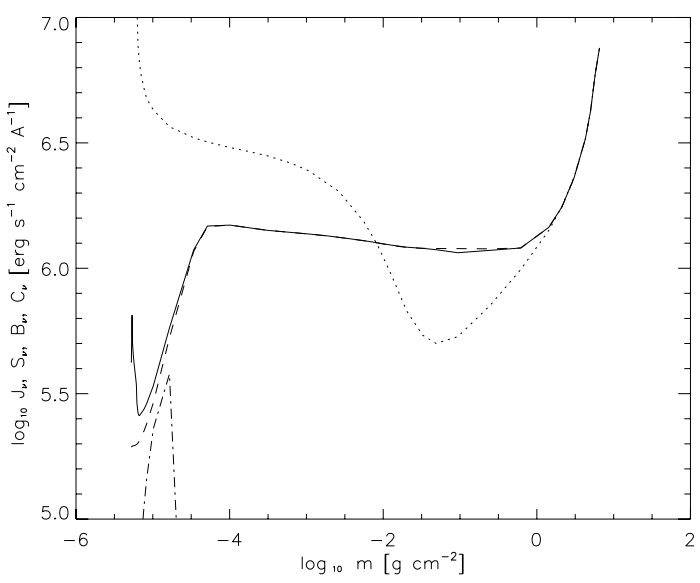

Fig. 7. Same as in Fig. 6 but for our NOS model. Compared to Fig. 6 the peak of the contribution function moved to deeper layers in the atmosphere.

\section{Conclusions}

Using the VAL model as an example of a classical 1D chromospheric model we found that the inclusion of LTE background lines does not lead necessarily to better i. e. more realistic results. The LTE emission lines are always over-predicted in strength in chromospheric models, which can lead to unrealistic high pumping effects between LTE lines and the hydrogen ionisation rate and therefore gives a wrong hydrogen ionisation balance. These pumping effects are very sensitive to the number of LTE lines sampled in the Lyman continuum. Therefore the wavelength grid on which the LTE background opacity is computed can influence the outcome of the simulations. Sampling of $0.02 \AA$ in this region showed that oxygen, nitrogen and sulfur have the most important influence on the hydrogen lines, while argon shows also some influence.

Moreover, our calculations of the model with 82 species computed in NLTE showed that the hydrogen spectra including LTE background opacity is still not identical to the same model without LTE background lines. For additional treatment of Ar IV in NLTE the difference between models with LTE background lines and without decrease further. Unfortunately, we are not able to include other ionisation stages of argon in the NLTE calculations, due to a lack of atomic data and hence, the question, if an inclusion of all important ionisation stages of argon 
in the NLTE calculations would remove the differences remains unsettled at the moment. Since PHOENIX cannot treat a height dependent $\varepsilon$ at the moment and a pure LTE treatment of the background lines is unrealistic, for the chromospheric part of the calculations it should be better not to include LTE background lines, though they are clearly useful for the photospheric description.

Since even the models with large NLTE sets (LAR, LARN, LAA, LAAN) do not reproduce the data as well as the original VAL model did, we conclude that the inclusion of large NLTE sets demands a change in the temperature structure of the chromospheric model and/or point to more general modelling problems with static $1 \mathrm{D}$ models that cannot account for horizontal and temporal chromospheric inhomogeneities. A study on how the temperature structure of the VAL model has to be altered to give a good fit of the data again (and if this is possible at all) is beyond the scope of this paper.

In general our calculations show the high impact of TR lines on the hydrogen emission lines for solar 1D chromospheric models and stress the necessity of a NLTE treatment especially for oxygen, nitrogen and sulfur lines. This impact of the highly ionised species - present only in the TR - has not been accounted for in previous chromospheric models. It clearly shows the need for a more realistic inclusion of the transition region in chromospheric models.

Acknowledgements. The model computations were performed at the Norddeutscher Verbund für Hoch- und Höchstleistungsrechnen (HLRN), at the Hamburger Sternwarte Apple G5 cluster financially supported by HBFG, and at St. Mary's University, Halifax, Canada. B. Fuhrmeister acknowledges financial support by the Deutsche Forschungsgemeinschaft under DFG SCHM 1032/16-1 and from Deutscher Akademischer Austauschdienst (DAAD).

\section{References}

Anderson, L. S. 1989, ApJ, 339, 558

Athay, R. G., \& Judge, P. G. 1995, ApJ, 438, 491

Ayres, T. R., \& Testerman, L. 1981, ApJ, 245, 1124

Carlsson, M., \& Judge, P. G. 1993, ApJ, 402, 344

Carlsson, M., \& Stein, R. F. 1997, ApJ, 481, 500

Carlsson, M., \& Stein, R. F. 2002, ApJ, 572, 626

Curdt, W., Brekke, P., Feldman, U., et al. 2001, A\&A, 375, 591

Fontenla, J. M., Avrett, E. H., \& Loeser, R. 1990, ApJ, 355, 700

Fuhrmeister, B., Schmitt, J. H. M. M., \& Hauschildt, P. H. 2005, A\&A, 439, 1137

Grevesse, N., \& Noels, A. 1993, in Origin and Evolution of the Elements, ed. N. Prantos, E. Vangioni-Flam, \& M. Casse (Cambridge University Press), 14

Harper, G. M. 1992, MNRAS, 256, 37

Hauschildt, P. H. 1993, JQSRT, 50, 301

Hauschildt, P. H., Allard, F., \& Baron, E. 1999, ApJ, 512, 377

Hauschildt, P. H., \& Baron, E. 1995, JQSRT, 54, 987

Hubeny, I., \& Lites, B. W. 1995, ApJ, 455, 376

Kurucz, R. L., \& Bell, B. 1995, Atomic line list (Kurucz CD-ROM, Cambridge, MA: Smithsonian Astrophysical Observatory)

Magain, P. 1986, A\&A, 163, 135

Mauas, P. J. D., \& Falchi, A. 1994, A\&A, 281, 129

McMurry, A. D., Jordan, C., \& Carpenter, K. G. 1999, MNRAS, 302, 48

Neckel, H. 1999, Sol. Phys., 184, 421

Short, C. I., \& Doyle, J. G. 1998, A\&A, 336, 613

Short, C. I., \& Hauschildt, P. H. 2005, ApJ, 618, 926

Vernazza, J. E., Avrett, E. H., \& Loeser, R. 1973, ApJ, 184, 605

Vernazza, J. E., Avrett, E. H., \& Loeser, R. 1981, ApJS, 45, 635

Wedemeyer, S., Freytag, B., Steffen, M., Ludwig, H.-G., \& Holweger, H. 2004, A\&A, 414, 1121

Wiedemann, G., Ayres, T. R., Jennings, D. E., \& Saar, S. H. 1994, ApJ, 423, 806

Young, P. R., Del Zanna, G., Landi, E., et al. 2003, ApJS, 144, 135 